\title{
A NEW METHOD OF DETERMINING \\ THE DIFFERENTIAL PARAMETERS AND INVARIANTS OF QUADRATIC DIFFERENTIAL QUANTTICS*
}

BY

\section{HEINRICH MASCHKE}

In the following article I propose to exhibit in a preliminary way a symbolic method, in close analogy with the symbolism used in the algebraic theory of invariants, for the construction and investigation of invariants of quadratic differential quantics. The method proves to be fully as successful as in algebra, the chief advantage lying in the fact that after the establishment of the fundamental principles of the method further reference to the formulas of transformation becomes unnecessary. $\dagger$

\section{§1. Introduction.}

If in the binary quadratic differential quantic

$$
A=\sum_{i, k=1}^{2} a_{i k} d x_{i} d x_{k}, \quad \quad\left(a_{k i}=a_{i k}\right),
$$

where the coefficients $a_{i k}$ are functions of $x_{1} x_{2}$, we introduce new variables $y_{1} y_{2}$ by the equations :

$$
x_{i}=x_{i}\left(y_{1}, y_{2}\right)
$$$$
(i=1,2) \text {, }
$$

then $A$ goes into the new expression :

$$
A=A^{\prime}=\sum a_{i k}^{\prime} d y_{i} d y_{k} .
$$

Let now $u, v, \cdots$ be arbitrary functions of $x_{1} x_{2}$, and denote by $u^{\prime}, v^{\prime}, \cdots$ the same functions after the substitution (2) has been made. Let further $\Omega$ be a function of the coefficients $a_{i k}$ and their derivatives and of $u, v, \ldots$ and their derivatives, and let $\Omega^{\prime}$ be the expression into which $\Omega$ is transformed, if the old quantities $a_{i k}, u, v, \cdots$ and their derivatives with respect to $x_{1} x_{2}$ are replaced

* Presented to the Society (Chicago) April 14, 1900. Received for publication April 5, 1900.

$\dagger$ A similar remark applies to the method followed by Mr. HessenBerg in his paper, Über die Invarianten linearer und quadratischer binärer Differentialformen und ihre Anwendung auf die Deformation der Flächen, Acta Mathematica, vol. 23, p. 121, 1900. 
by the new quantities $a_{i k}^{\prime}, u^{\prime}, v^{\prime}, \ldots$ and their derivatives with respect to $y_{1} y_{2}$, where the $a_{i k}^{\prime}$ are defined by the equation (3). If $\Omega$ satisfies the equation :

$$
\Omega^{\prime}=\Omega,
$$

$\Omega$ is called an invariant of the quadratic differential quantic $A$.

In this sense every arbitrary function $u, v, \ldots$ of $x_{1} x_{2}$ is an invariant of $A$ on account of the equations : $u^{\prime}=u, v^{\prime}=v, \cdots$.

Invariants $\Omega$ which actually contain at least one arbitrary function are generally called differential parameters, while those which involve no arbitrary function are called differential invariants.

The most important differential parameters are the following :*

$$
\begin{aligned}
\Delta_{1} u & =\sum_{r, s} A_{r s} \frac{\partial u}{\partial x_{r}} \frac{\partial u}{\partial x_{s}}, \\
\nabla(u, v) & =\sum_{r, s} A_{r s} \frac{\partial u}{\partial x_{r}} \frac{\partial v}{\partial x_{s}}, \\
\Delta_{2} u & =\sum_{r, s} A_{r s} \frac{\partial^{2} u}{\partial x_{r} \partial x_{s}}-\sum_{r, s, i, k} A_{r s} A_{i k}\left[\begin{array}{c}
r s \\
k
\end{array}\right] \frac{\partial u}{\partial x_{k}} .
\end{aligned}
$$

Here $A_{i k}$ denotes the minor of the element $a_{i k}$ in the determinant

$$
D=\left|a_{i k}\right|
$$

divided by the determinant $D$ (which is always supposed to be different from zero). Further :

$$
\left[\begin{array}{c}
r s \\
k
\end{array}\right]=\frac{1}{2}\left(\frac{\partial a_{r k}}{\partial x_{s}}+\frac{\partial a_{s k}}{\partial x_{r}}-\frac{\partial a_{r s}}{\partial x_{k}}\right)
$$

is the so-called triple index symbol introduced by CHRISTOFFEL. $\dagger$

The transformed expression $A^{\prime}$ (3) being obtained from (1) by linear substitution of the differentials, the connection between the $a_{i k}^{\prime}$ and the $a_{i k}$ will be the same as in the case of the transformation of an algebraic quantic with coefficients $a_{i k}$ and the quantities $\partial x_{i} / \partial y_{k}$ as coefficients of the linear substitution.

Hence we have

$$
D^{\prime}=r^{2} D
$$

where

$$
r=\left|\frac{\partial x_{i}}{\partial y_{k}}\right| \quad(i, k=1,2) .
$$

* Cf. BranchI, Vorlesungen über Differentialgeometrie, Leipzig, 1899, pp. 41 and 47.

†Über die Transformation der homogenen Differentialausdrïcke zweiten Grades, Crelle's J o u rnal, vol. 70, p. 48, formula (4). 
If now $F$ is an invariant of $A$, then

$$
\frac{\partial F^{\prime \prime}}{\partial y_{1}} d y_{1}+\frac{\partial F^{\prime \prime}}{\partial y_{2}} d y_{2}=\frac{\partial F}{\partial x_{1}} d x_{1}+\frac{\partial F}{\partial x_{2}} d x_{2} ;
$$

and if $\Phi$ is also an invariant, we have :

$$
\frac{\partial F^{\prime}}{\partial y_{1}} \frac{\partial \Phi^{\prime}}{\partial y_{2}}-\frac{\partial F^{\prime}}{\partial y_{2}} \frac{\partial \Phi^{\prime}}{\partial y_{1}}=r\left(\frac{\partial F}{\partial x_{1}} \frac{\partial \Phi}{\partial x_{2}}-\frac{\partial F}{\partial x_{2}} \frac{\partial \Phi}{\partial x_{1}}\right)
$$

With respect to any functions $U$ and $V$ of $x_{1} x_{2}$ let us introduce the following abbreviations :

$$
\begin{gathered}
\frac{\partial U}{\partial x_{i}}=U_{i}, \quad \frac{\partial^{2} U}{\partial x_{i} \partial x_{k}}=U_{i k}, \quad \text { etc. } ; \\
U_{1} V_{2}-U_{2} V_{1}=(U, V) .
\end{gathered}
$$

Further, with respect to a transformation from the variables $x_{1} x_{2}$ to the variables $y_{1} y_{2}$ we use the abbreviation :

$$
\frac{1}{\sqrt{D}}=a
$$

Then from (12) and (10)'we have in these notations :

$$
a^{\prime}(F, \Phi)^{\prime}=a(F, \Phi),^{*}
$$

and accordingly the fundamental theorem :

Theorem I. If $F$ and $\Phi$ are invariants of $A$ then $a(F, \Phi)$ is again an invariant of $A$.

\section{§2. The Symbolic Method.}

Let now $f$ be a (symbolic) function of $x_{1} x_{2}$, and $f_{1}$ and $f_{2}$ its two derivatives: then on setting

$$
f_{i} f_{k}=a_{i k},
$$

we may consider the expression $\left(f_{1} d x_{1}+f_{2} d x_{2}\right)^{2}$ as symbolically equal to $A$. In this case we shall say for shortness that $f$ is a symbol of $A$.

If $f, \phi, \ldots$ are several symbols of $A$, since $f^{\prime}=f, \phi^{\prime}=\phi, \cdots$, these symbols are in the first place invariants of $A$. Building up now according to theorem I other invariants out of these and of arbitrary functions $u, v, \cdots$ and forming products with these symbolic invariants as factors, we have at once by the same reasoning as in algebra an actual invariant of $A$ before us, in case every

\footnotetext{
* [In subsequent papers it would be desirable to introduce for $a(F, \Phi)$ the notation $\{F, \Phi\}$.]
} 
symbol $f, \phi, \ldots$ appears precisely twice and in such a way that only the connections $f_{i} f_{k}, \phi_{i} \phi_{k}, \cdots$ occur. (We shall presently see, however, that the last restriction can be partly removed.)

Thus the expression $a^{2}(f, u)^{2}$ satisfies the conditions just stated. Indeed we have

$$
\begin{aligned}
a^{2}(f, u)^{2} & =\frac{1}{D}\left(f_{1}^{2} u_{2}^{2}-2 f_{1} f_{2} u_{1} u_{2}+f_{2}^{2} u_{1}^{2}\right)=\frac{1}{D}\left(a_{11} u_{2}^{2}-2 a_{12} u_{1} u_{2}+a_{22} u_{1}^{2}\right) \\
& =A_{11} u_{1}^{2}+2 A_{12} u_{1} u_{2}+A_{22} u_{2}^{2}=\sum A_{r s} \frac{\partial u}{\partial x_{r}} \frac{\partial u}{\partial x_{s}}=\Delta_{1} u,
\end{aligned}
$$

that is,

Likewise we obtain :

$$
a^{2}(f, u)^{2}=\Delta_{1} u
$$

From (17) there follows :

$$
a^{2}(f, u)(f, v)=\nabla(u, v) .
$$

and similarly :

$$
\frac{\partial a_{i k}}{\partial x_{l}}=f_{i} f_{k l}+f_{k} f_{i l}
$$

$$
\begin{aligned}
& \frac{\partial a_{k l}}{\partial x_{i}}=f_{k} f_{l i}+f_{l} f_{k i}, \\
& \frac{\partial a_{l i}}{\partial x_{k}}=f_{l} f_{i k}+f_{i} f_{l k} .
\end{aligned}
$$

Hence we have the symbolic form :

$$
f_{i} f_{k l}=\frac{1}{2}\left(\frac{\partial a_{i k}}{\partial x_{l}}+\frac{\partial a_{i l}}{\partial x_{k}}-\frac{\partial a_{k l}}{\partial x_{i}}\right)=\left[\begin{array}{c}
k l \\
i
\end{array}\right]
$$

of the triple index symbol.

We see therefore that, the other conditions given above being satisfied, we can also admit the connections $f_{i} f_{k l}$ as well as the connections $f_{i} f_{k}$.

For example, from theorem I we see that the expression $a(f, a(f, u))$ is formally invariant; it also satisfies the conditions for actual existence. Indeed we find

$$
a(f, a(f, u))=a^{2}\left[f_{1}\left(f_{12} u_{2}-f_{22} u_{1}\right)-f_{2}\left(f_{11} u_{2}-f_{12} u_{1}\right)\right]+\cdots
$$

The complete computation leads to the result :

$$
a\left(f, a\left(f_{1} u\right)\right)=\Delta_{2} u \text {. }
$$

I give the values of some other symbolic expressions :

$$
a^{3}(f, u)(\phi, u)(f, a(\phi, u))=\frac{1}{2} \nabla\left(u, \Delta_{1} u\right),
$$




$$
\begin{aligned}
& a^{4}(\phi, u)(\psi, u)(f, a(\phi, u))(f, a(\psi, u))=\frac{1}{4} \Delta_{1}\left(\Delta_{1} u\right), \\
& a^{2}(f, \phi)(a(f, u), a(\phi, u))=2 \Delta_{22} u, * \\
& a^{2}(f, a(\phi, u))(\phi, a(f, u))=\Delta_{2}^{2} u-2 \Delta_{22} u, \\
& (f, \phi)(f, a(\phi, u))=0 .
\end{aligned}
$$

For the computation with symbolic expressions the following identity is useful :

$$
(a, b)(c, d)+(a, c)(d, b)+(a, d)(b, c)=0 .
$$

Also the relation (25) (which becomes an identity only when actual values are substituted for the symbols) often serves to reduce complicated expressions.

As an example the invariant $\Delta_{1} u \cdot \Delta_{1} v-\nabla^{2}(u, v)$ will be transformed. We have

$$
\begin{aligned}
\Delta_{1} u \cdot \Delta_{1} v-\nabla^{2}(u, v) & =a^{4}\left[(f, u)^{2}(\phi, v)^{2}-(f, u)(f, v)(\phi, u)(\phi, v)\right] \\
& =a^{4}(f, u)(\phi, v)[(f, u)(\phi, v)-(f, v)(\phi, u)] .
\end{aligned}
$$

The quantity in the bracket reduces by means of $(26)$ to $(f, \phi)(u, v)$. Hence

$$
\Delta_{1} u \cdot \Delta_{1} v-\nabla^{2}(u, v)=a^{4}(f, \phi)(f, u)(\phi, v)(u, v) .
$$

If to the right side of this equation we add the expression obtained from it by interchanging the equivalent symbols $f, \phi$ and divide by 2 , we obtain :

Hence :

$$
\frac{1}{2} a^{4}(f, \phi)(u, v)[(f, u)(\phi, v)-(f, v)(\phi, u)] .
$$

$$
\Delta_{1} u \cdot \Delta_{1} v-\nabla^{2}(u, v)=\frac{1}{2} a^{4}(f, \phi)^{2}(u, v)^{2}=\frac{1}{D}(u, v)^{2} .
$$

As a second example of symbolic computation I give a proof of the formula :

$$
2 \Delta_{2} u \cdot \nabla\left(u, \Delta_{1} u\right)-\Delta_{1}\left(\Delta_{1} u\right)=4 \Delta_{1} u \cdot \Delta_{22} u \cdot \dagger
$$

We have from $(21),(22),(23)$ :

$$
\begin{aligned}
& 2 \Delta_{2} u \cdot \nabla\left(u, \Delta_{1} u\right)-\Delta_{1}\left(\Delta_{1} u\right)= \\
& \quad 4 a^{4}(\phi, u)(f, a(\phi, u))[(f, u)(\psi, a(\psi, u))-(\psi, u)(f, a(\psi, u))] .
\end{aligned}
$$

* For the definition of $\Delta_{22} u$ see Branchr, loc. cit., p. 48.

†This formula is given by BIANCHI, loc. cit., p. 48 without proof. Compare also HessenBERG, loc. cit., p. 143. 
The application of (26) reduces the quantity in the brackets to

Again by (26) we obtain :

$$
(f, \psi)(u, a(\psi, u))
$$

$$
\begin{aligned}
(f, a(\phi, u))(u, a(\psi, u))=.(u, f) & (a(\psi, u), a(\phi, u)) \\
+ & (f, a(\psi, u))(u, a(\phi, u)) .
\end{aligned}
$$

The second term of this sum vanishes on account of (25) if we multiply by $(\phi, u)(f, \psi)$, and now we have :

$2 \Delta_{2} u \cdot \nabla\left(u, \Delta_{1} u\right)-\Delta_{1}\left(\Delta_{1} u\right)=-4 a^{4}(f, u)(\phi, u)(f, \psi)(a(\psi, u), a(\phi, u))$.

Interchanging here the equivalent symbols $\phi, \psi$, adding the resulting expression to the right side, and dividing by 2 , we obtain on the right side the factor $(\phi, u)(f, \psi)-(\psi, u)(f, \phi)$ which reduces, according to $(26)$, to the product $(\phi, \psi)(f, u)$. The desired result is now obtained :

$$
\begin{aligned}
2 \Delta_{2} u \cdot \nabla\left(u, \Delta_{1} u\right)-\Delta_{1}\left(\Delta_{1} u\right) & =2 a^{4}(f, u)^{2}(\phi, \psi)(a(\phi, u), a(\psi, u)) \\
& =4 \Delta_{1} u \cdot \Delta_{22} u .
\end{aligned}
$$

The symbolic expressions for the second derivatives of $a_{i k}$ appear as aggregates of the form $f_{i k} f_{l m}$ and $f_{i} f_{k l m}$. But it is impossible to express conversely each of these two quantities separately in terms of the $a_{i k}$ and their derivatives. There exist only certain connections of the $f_{i k} f_{l m}$ and $f_{i} f_{k l m}$ which admit of such a representation, e. g.,

$$
f_{11} f_{22}-f_{12}^{2}=\frac{1}{2}\left(\frac{\partial^{2} a_{11}}{\partial x_{2}^{2}}-2 \frac{\partial^{2} a_{12}}{\partial x_{1} \partial x_{2}}+\frac{\partial^{2} a_{22}}{\partial x_{1}^{2}}\right)
$$

and others. A similar remark holds good also for the case of all the higher derivatives of the $a_{i k}$.

The most important example of this kind is

$$
a^{2}(\phi, \psi)(a(f, \phi), a(f, \psi))
$$

Derivatives of $f$ of order $k$ higher than the second do not occur, but the terms which are quadratic in the $f_{i k}$ are all contained in the expression

$$
((f, \phi),(f, \psi))
$$

an expression which can also be written in this form :

$$
\frac{\partial(f, \phi)(f, \psi)_{2}}{\partial x_{1}}-\frac{\partial(f, \phi)(f, \psi)_{1}}{\partial x_{2}},
$$


and here in the expressions which are to be differentiated, i. e., in $(f, \phi)(f, \psi)_{i}$ the coefficients $f$ occur only in the connection $f_{i} f_{k l}$. Therefore the above symbolic invariant represents an actual invariant of $A$, and since it does not contain arbitrary functions, a differential invariant. The result is indeed :

$$
a^{2}(\phi, \psi)(a(f, \phi), a(f, \psi))=2 K
$$

where $K$ is the expression* for the curvature of $A$. In passing we derive by comparison with (24) the formula:

$$
K=\Delta_{22}(f)
$$

\section{§3. Covariants.}

The complete differential of every invariant represents immediately a linear covariant of $A$. Covariants of higher degrees are therefore obtained at once by forming products of these symbolic linear covariants and (if necessary) also invariants, in such a way that every symbol $f, \phi, \ldots$ occurs precisely twice and so that the quadratic terms of the derivatives of the symbols admit of an actual interpretation in terms of the $a_{i k}$ and their derivatives.

The simplest quadratic covariant is :

$$
\left(\frac{\partial a(f, u)}{\partial x_{1}} d x_{1}+\frac{\partial a(f, u)}{\partial x_{2}} d x_{2}\right)\left(f_{1} d x_{1}+f_{2} d x_{2}\right)
$$

This covariant is however irrational because the quantity a occurs only once as a factor. The simplest rational quadratic covariant is the following :

$$
a(f, \phi)\left(\frac{\partial a(f, u)}{\partial x_{1}} d x_{1}+\frac{\partial a(f, u)}{\partial x_{2}} d x_{2}\right)\left(\phi_{1} d x_{1}+\phi_{2} d x_{2}\right) ;
$$

it is identical with a covariant which BIANCHI $\dagger$ calls the second covariant differential of $u$.

As a further example I mention :

$2 a(f, \phi)\left(\frac{\partial a(f, F)}{\partial x_{1}} d x_{1}+\frac{\partial a(f, F)}{\partial x_{2}} d x_{2}\right)\left(\phi_{1} d x_{1}+\phi_{2} d x_{2}\right)\left(F_{1} d x_{1}+F_{2} d x_{2}\right)$, a cubic simultaneous covariant of $A$ and $B=\sum b_{i k} d x_{i} d x_{k}$, where $f, \phi$ are symbols of $A$, and $F$ is a symbol of $B$. This is one of the cubic covariants mentioned by Bianchi, loc. cit., p. 55, and by KNoвladch, loc. cit., p. 197.

\footnotetext{
* See Bianchi, loc. cit., pp. 50-53, and KNoblaUch, Einleitung in die Theorie der krummen Flächen, Leipzig, 1888, pp. 174-179.

† loc. cit., p. 46.
} 


\section{§4. Differential quantics in general.}

The preceding deductions can be extended without much difficulty from the case of binary differential quantics to those of $n$ variables (and also from quadratic quantics to those of higher orders). So we have at once for ternary quantics :

$$
\begin{aligned}
\Delta_{1} u & =\frac{1}{2} a^{2}(f, \phi, u)^{2}, \\
\nabla(u, v) & =\frac{1}{2} a^{2}(f, \phi, u)(f, \phi, v), \\
\Delta_{2} u & =\frac{1}{2} a(f, \phi, a(f, \phi, u)),
\end{aligned}
$$

and here the generalization for $n$ variables is evident.

The invariant forms which are analogous to the curvature are in the ternary case :

$$
a^{2}(\phi, \psi, \chi)(a(f, \phi, \psi), a(f, \chi, \omega), \omega),
$$

with $f, \phi, \psi, \chi, \omega$ as equivalent symbols, and in the general case :

$a^{2}\left(\phi^{1}, \phi^{2}, \ldots \phi^{n}\right)\left(a\left(f, \phi^{1}, \phi^{2}, \cdots \phi^{n-1}\right), a\left(f, \phi^{n}, \psi^{1}, \psi^{2}, \cdots \psi^{n-2}\right), \psi^{1}, \psi^{2}, \cdots \psi^{n-2}\right)$, with $f, \phi^{1}, \phi^{2}, \cdots \phi^{n}, \psi^{1}, \psi^{2}, \ldots \psi^{n-2}$ as equivalent symbols. It is easily seen that here the terms quadratic in $f_{i k}$ occur only in the connection :

$$
f_{i l} f_{k m}-f_{i m} f_{k l}=\frac{1}{2}\left(\frac{\partial^{2} a_{i l}}{\partial x_{k} \partial x_{m}}+\frac{\partial^{2} a_{k m}}{\partial x_{i} \partial x_{l}}-\frac{\partial^{2} a_{i m}}{\partial x_{i} \partial x_{l}}-\frac{\partial^{2} a_{k l}}{\partial x_{i} \partial x_{m}}\right) .
$$

The University of Chicago, April, 1900. 